Service social

\title{
Santé psychologique des demandeurs d'asile dans un contexte politique défavorable : perspectives d'intervenants sociaux sur les réformes du système de protection des réfugiés
}

\section{Sandrine Sarradet}

Volume 61, numéro 1, 2015

Être reconnu et protégé

URI : https://id.erudit.org/iderudit/1033740ar

DOI : https://doi.org/10.7202/1033740ar

Aller au sommaire du numéro

Éditeur(s)

École de service social de l’Université Laval

ISSN

1708-1734 (numérique)

Découvrir la revue

Citer cet article

Sarradet, S. (2015). Santé psychologique des demandeurs d'asile dans un contexte politique défavorable : perspectives d'intervenants sociaux sur les réformes du système de protection des réfugiés. Service social, 61(1), 57-75. https://doi.org/10.7202/1033740ar
Résumé de l'article

Ce projet de recherche empirique visait à explorer les conséquences des réformes fédérales du système de protection des réfugiés sur la santé psychologique des demandeurs d'asile à Montréal, notamment à travers l'offre de services d'aide à cette population. Les intervenants sociaux interrogés on fait ressortir plusieurs manières dont ces réformes accentuent les difficultés post-migratoires des demandeurs d'asile, notamment par l'insuffisance des ressources d'aide psychosociale et le discours anti-réfugié véhiculé dans la population. Ces résultats reflètent la nécessité d'investir adéquatement dans les ressources d'aide à cette population et de promouvoir le développement, dans la société d'accueil, d'une vision respectueuse de la réalité et des droits des demandeurs d'asile. 


\title{
SANTÉ PSYCHOLOGIQUE DES DEMANDEURS D'ASILE DANS UN CONTEXTE POLITIQUE DÉFAVORABLE : PERSPECTIVES D'INTERVENANTS SOCIAUX SUR LES RÉFORMES DU SYSTĖME DE PROTECTION DES RÉFUGIÉS 1
}

\author{
SARRADET, Sandrine
}

\begin{abstract}
RÉSUMÉ
Ce projet de recherche empirique visait à explorer les conséquences des réformes fédérales du système de protection des réfugiés sur la santé psychologique des demandeurs d'asile à Montréal, notamment à travers l'offre de services d'aide à cette population. Les intervenants sociaux interrogés ont fait ressortir plusieurs manières dont ces réformes accentuent les difficultés post-migratoires des demandeurs d'asile, notamment par l'insuffisance des ressources d'aide psychosociale et le discours anti-réfugié véhiculé dans la population. Ces résultats reflètent la nécessité d'investir adéquatement dans les ressources d'aide à cette population et de promouvoir le développement, dans la société d'accueil, d'une vision respectueuse de la réalité et des droits des demandeurs d'asile.
\end{abstract}

Mots clés : demandeur d'asile, santé psychologique, ressources communautaires, système de protection des réfugiés, programme fédéral de santé intérimaire, discours anti-réfugié.

\begin{abstract}
This empirical research project aimed to understand the effects of the refugee protection system's federal reforms on asylum seekers' psychological well-being, particularly through help services provided to this population. Interviewed stakeholders have identified several ways in which these reforms might accentuate asylum seekers' post-migration difficulties, including the lack of psychosocial support resources and the anti-refugee discourse amongst the population. These results show the need to promote awareness of the reality of asylum seekers as well as to foster consideration for their rights within the host society.
\end{abstract}

Key words : asylum seeker, psychological well-being, community resources, refugee protection system, interim federal health program, anti-refugee discourse.

1. Cet article est inspiré du projet d'études indépendant intitulé Adaptation psychologique post-migratoire des demandeurs d'asile dans un contexte politique défavorable: perspectives d'intervention, supervisé par Dre Jill Hanley $(\mathrm{PhD})$ dans le cadre de la maîtrise en travail social à l'Université McGill. 


\section{INTRODUCTION}

Dans les dernières décennies, plusieurs facteurs tels que la pauvreté, l'instabilité, la guerre et la mondialisation, ont fait augmenter la migration et la fuite de personnes hors de leur pays d'origine (Cemlyn et Briskman, 2003). Le Haut Commissariat des Nations unies pour les réfugiés² (HCR) définit un réfugié comme une personne incapable de retourner dans son pays d'origine et forcée de résider ailleurs que dans ce dernier. Cette situation doit découler d'une crainte fondée de persécution sur la base de la race, la nationalité, la religion, l'opinion politique ou l'appartenance à un groupe social, sans perspective raisonnable de solution permanente (CISR, 2006). En effet, selon la Convention de Genève (1951), signée par plusieurs pays dont le Canada, le principe de non-refoulement stipule qu'« aucun État n'expulsera ou ne refoulera, de quelque manière que ce soit, un réfugié vers un pays où il risque la persécution ».

Les demandeurs d'asile sont des personnes ayant fuit leur pays vers un autre territoire, fait une demande de statut de réfugié dans ce territoire et en attente de décision par rapport à leur application (Crumlish et O'Rourke, 2010). Au Canada, on estimait à plus de 24900 le nombre de demandeurs d'asile en 2011 (CIC, 2012b). Le taux d'acceptation des demandeurs d'asile aurait été d'environ $40 \%$ entre 2008 et 2010 (ASFC, 2010). Le processus de demande d'asile est complexe, parsemé de difficultés potentielles dans la société d'accueil, notamment sur le plan psychologique. Parmi celles-ci, on retrouve le morcellement identitaire (Peterson, 2012), ainsi que le désespoir relié à la perte d'un sens de la continuité et à l'incertitude concernant l'avenir (Lacroix, 2004a). Les demandeurs d'asile semblent également particulièrement à risque de développer certaines problématiques de santé mentale, notamment l'état de stress posttraumatique (Crumlish et O'Rourke, 2010 ; George, 2010), la dépression et les troubles anxieux (Gladden, 2012). Cela dit, la question de la santé mentale dans cette population est controversée et l'interprétation des problématiques doit tenir compte de nombreux facteurs culturels et sociaux (Lacroix et Sabbah, 2011). Les difficultés apparaissent dans l'ensemble des sphères où le demandeur d'asile évolue dans son parcours post-migratoire et sont susceptibles d'être aggravées par des facteurs tels que l'incertitude par rapport au statut, la restriction dans l'accès aux ressources et la crainte de déportation (Masocha et Simpson, 2011).

Dans les dernières années, les orientations idéologiques et les politiques sociales du gouvernement canadien par rapport aux demandeurs d'asile ont semblé peu favorables à l'adaptation post-migratoire de cette population dans la société d'accueil. D'abord, les idéologies économiques dominantes dans l'ensemble des pays industrialisés conceptualisent les relations entre êtres humains comme régies par la compétition pour des ressources limitées (Okipikti et Aymer, 2003). Dans le cas des demandeurs d'asile au Canada, cette conception se traduit notamment par l'évaluation de leur légitimité sur le territoire canadien en termes de fardeau financier et de menace pour la sécurité et la prospérité économique du pays.

Cette idéologie qui domine la pensée conservatrice s'est d'abord traduite, dès l'arrivée au pouvoir du Parti conservateur comme gouvernement minoritaire en 2006, par un discours antiréfugiés laissant sous-entendre que ces derniers étaient des fraudeurs voulant abuser du

2. Dans l'ensemble de l'article, l'utilisation du genre masculin a été adoptée afin de faciliter la lecture et n'a aucune intention discriminatoire. 
système canadien (Cemlyn et Briskman, 2003 ; Cambridge et Williams, 2004). Cette rhétorique a par la suite servi au gouvernement à justifier les réformes que l'obtention d'un statut majoritaire en mai 2011 lui a permis de mettre en œuvre.

En effet, plusieurs modifications au système canadien d'octroi de l'asile et aux services offerts aux demandeurs ont été mises en place dans les dernières années. Parmi ces modifications, on retrouve le projet de loi C-31. D'une part, celui-ci se traduit par l'instauration d'une liste de pays d'origine désignés (POD) considérés comme «sûrs », à l'égard desquels le gouvernement suppose qu'une demande d'asile est moins légitime. De plus, on retrouve une accélération du processus de demande d'asile qui se traduit entre autres par la réduction des délais avant l'audience (CIC, 2012b) ${ }^{3}$.

Différentes instances œuvrant de près ou de loin auprès des demandeurs d'asile ont formulé plusieurs critiques du projet de loi. D'abord, tel qu'exprimé par l'Association canadienne des libertés civiles, on déplore le revirement idéologique du Canada envers les réfugiés, considérant que celui-ci passe « d'un rôle de protecteur des droits humains à celui de contrevenant aux droits de la personne »(Des Rosiers et Mendelsohn Aviv, 2012). De plus, des préoccupations importantes ressortent par rapport à l'équité du traitement des demandes, considérant que les ressortissants des POD voient leur demande traitée différemment (Association du Barreau canadien, 2012). En outre, dans plusieurs de ces pays, des problèmes sérieux de persécution, de discrimination et de violence contre des individus sont recensés, souvent avec la participation des représentants de l'État (Conseil canadien pour les réfugiés, 2012).

Des craintes sont également émises quant à la réduction considérable des délais dans le processus de demande d'asile, tel qu'exprimé dans le mémoire du Barreau du Québec (2012) :

Les personnes qui présentent une demande d'asile à un port d'entrée auront 15 jours pour produire le document. Si le demandeur omet des renseignements, des conclusions défavorables pourraient en être tirées par la suite. Les demandeurs d'asile souffrent parfois du trouble de stress post-traumatique ou sont confrontés à des obstacles culturels ou sexuels les empêchant de communiquer pleinement leur crainte de la persécution à un agent de CIC ou de l'ASFC. Les demandeurs d'asile auront ensuite leur audience dans un délai de 30 à 60 jours. Ce n'est pas suffisant pour permettre aux demandeurs de retenir un avocat et préparer leur dossier. Les demandeurs doivent souvent obtenir des documents de leur pays d'origine, puis les faire traduire avant de les déposer. Dans de nombreux cas, des témoins doivent aussi être retenus, comme des psychologues ou des médecins.

La distinction entre réfugiés légitimes et illégitimes renforce non seulement la marginalisation de ceux issus de la dernière catégorie, mais sert également à justifier des politiques restrictives à leur égard comme le reflète la réforme du Programme fédéral de santé intérimaire mise en place en juin 2012. En plus de restrictions à l'accès aux soins de santé de base pour les

3. Bien que le projet de loi C-31 implique davantage d'éléments que ceux décrits dans cet article, nous nous sommes concentrés sur l'établissement des POD et la réduction des délais avant l'audience, aspects principaux identifiés par les participants. 
personnes provenant des POD, le gouvernement canadien ne couvre plus certains soins offerts à l'ensemble des demandeurs d'asile, notamment les services psychologiques (CIC, 2012a).

La logique dans laquelle s'inscrivait cette réforme est bien représentée par un communiqué transmis par l'ancien ministre de la Citoyenneté et de l'Immigration Jason Kenney lors de son annonce :

Cette réforme élimine également un facteur d'attraction pour les personnes qui pourraient avoir envie de présenter une demande d'asile non fondée au Canada. Elle nous permet à la fois de protéger la santé et la sécurité publiques, de veiller à ce que l'argent des contribuables soit dépensé judicieusement et de préserver l'intégrité de notre système d'immigration (CIC, 2012a).

En effet, comme les demandes des personnes issues des POD sont considérées comme illégitimes en territoire canadien, il devient justifié pour le gouvernement conservateur de restreindre les droits de ces personnes aux soins de santé de base pour les décourager de «profiter du système », mais également dans une optique de réduction des dépenses pour le Canada.

À ce propos, nombreuses sont les inquiétudes émises par les acteurs du milieu de la santé, entre autres, quant à l'impact des coupures des soins de santé sur certains groupes de demandeurs d'asile. Dans une décision rendue le 4 juillet 2014 dans l'affaire Médecins canadiens pour les soins aux réfugiés c. Canada, la Cour fédérale concluait que les modifications apportées au Programme fédéral de santé intérimaire (PFSI) en juin 2012 « ont manifestement eu de graves répercussions sur la santé physique et le bien-être psychologique de nombreuses personnes » et qu'elles constituaient un « traitement cruel et inusité » qui violait la Charte canadienne des droits et libertés ${ }^{4}$. Au niveau des soins psychologiques qui ne sont toujours pas couverts, on déplore le fait que les demandeurs d'asile ne puissent avoir accès aux services adéquats considérant la prévalence de problèmes de santé mentale dans cette population (Médecins du Monde, 2015).

À la lumière de ces renseignements, il nous semble que les idéologies politique et économique dominantes actuelles, de même que les lois et les politiques sociales qui en découlent, peuvent avoir un impact important sur la santé psychologique des demandeurs d'asile au Canada. Dans le cadre de cet article, la santé psychologique s'entend comme l'optimisation du sentiment subjectif de bien-être psychologique à travers le processus migratoire. Cette définition s'inspire notamment de celle de l'Organisation mondiale de la santé (OMS) quant à la santé mentale, définie comme un « état de bien-être dans lequel l'individu réalise ses propres capacités, peut faire face aux tensions ordinaires de la vie, et est capable de contribuer à sa communauté » (Vignal et Geny-Benkovichi, 2012). De plus, Rees (2003, dans Bernhard et al., 2007) définit la

4. À compter du 5 novembre 2014, le PFSI a été partiellement rétabli pour les demandeurs d'asile issus des POD suite à la décision de la Cour fédérale en juillet 2014 (CIC, 2014). Comme le gouvernement interjette appel, l'évolution de la situation est à suivre de près dans les prochains mois. La couverture des soins psychologiques n'est toujours pas rétablie. 
notion de bien-être comme «un état holistique incluant un contentement psychologique, physique, spirituel, social et culturel » reflétant l'habileté à fonctionner dans la société.

Ainsi, nous avons tenté d'explorer, auprès d'intervenants en contact avec des demandeurs d'asile sur le terrain, en quoi ces changements affectaient les différentes sphères post-migratoires entourant les demandeurs d'asile, notamment à travers l'offre de services à cette population, en portant une attention particulière sur la santé psychologique. Nous avons recherché dans quelle mesure la manière dont les participants vivaient les changements à l'échelle de leur organisme et sur le territoire montréalais constituait la confirmation des craintes et des critiques exprimées dans la sphère publique suite aux réformes de 2012.

Dans un premier temps, nous décrirons la méthodologie utilisée, pour ensuite aborder les perspectives des participants sur les réformes. Ces résultats feront l'objet d'une discussion et seront finalement utilisés pour élaborer des recommandations sur les mesures à adopter pour favoriser la santé psychologique des demandeurs d'asile dans la société d'accueil.

\section{MÉTHODOLOGIE}

L'étude effectuée, de nature qualitative et exploratoire, a consisté en une série de huit entrevues auprès d'informateurs clés ${ }^{5}$ qui travaillaient auprès de demandeurs d'asile à Montréal, la plupart les côtoyant sur une base régulière dans l'offre de services psychosociaux de première ligne. Les entrevues ont eu lieu à l'automne 2013 , soit un peu plus d'un an après les réformes. La grande majorité des participants provenait du milieu communautaire, bien que le milieu institutionnel ait également été représenté par une personne. Les participants représentaient différentes ressources dont les services variaient : hébergement, accompagnement psychosocial, services psychologiques, aide pratique à l'intégration, soins de santé, assistance juridique. Ainsi, bien que les ressources ne soient généralement pas spécialisées dans l'offre de soutien psychologique sous forme de thérapie, elles jouent tout de même un rôle dans la promotion du bien-être psychologique des demandeurs d'asile après la migration. Certains participants faisaient également de la représentation politique pour les demandeurs d'asile à travers leur organisation. Plusieurs d'entre eux travaillaient pour des milieux offrant des services aux immigrants de manière générale qui accueillent également des demandeurs d'asile.

Les entrevues ont par la suite été transcrites et analysées selon la méthode de théorie ancrée. Elles ont été codées selon une grille de codage sélectif élaborée à partir des thèmes explorés dans le questionnaire. Des sous-thèmes récurrents ont ensuite été identifiés, pour en arriver à élaborer une description de la situation étudiée à travers un processus de codage axial. Les sousthèmes émergents ont donc été mis en relation pour élaborer des propositions et dresser un portrait des enjeux étudiés, selon les perceptions rencontrées (Creswell, 2013).

5. Bien qu'aucune ressource ou personne ne soit nommée, les intervenants provenant des ressources susceptibles d'être identifiées ont été avisés de l'intention de l'auteure de publier les résultats de la recherche. 


\section{Résultats : Perspectives des participants sur les enjeux reliés à la santé psychologique des demandeurs d'asile en lien avec le contexte politique}

À travers les entrevues, les participants ont eu l'occasion de s'exprimer sur plusieurs thèmes reliés à la santé psychologique des demandeurs d'asile dans le contexte politique actuel, et ce, à différentes étapes de leur cheminement dans la société d'accueil. D'abord, les intervenants rencontrés ont identifié les besoins qu'ils percevaient chez les demandeurs d'asile: développement d'un sentiment de sécurité, aide pratique par rapport aux besoins de base, création d'un réseau social, accompagnement dans le processus d'adaptation et accompagnement thérapeutique et psychologique dans plusieurs cas $^{6}$. Ils ont également décrit l'influence des changements politiques actuels sur le bien-être des demandeurs d'asile à Montréal, autant en ce qui concerne leur intégration dans la société d'accueil que dans la manière dont les ressources pouvaient répondre à leurs besoins. Ils ont ensuite abordé la situation actuelle des ressources d'aide aux demandeurs d'asile à Montréal. Finalement, ils ont exprimé des recommandations sur ce qui devait être développé pour améliorer la situation de cette population dans l'environnement post-migratoire.

\section{Critique de la loi C-31}

Les participants ont exprimé leur opinion sur la loi C-31, qui constitue un virage important effectué par le gouvernement conservateur quant au processus de demande d'asile. Cette distinction a entraîné divers changements dans le processus de demande d'asile, notamment quant aux délais avant l'audience et à l'accès aux services.

D'abord, l'établissement d'une liste de pays d'origine désignés est décrit par une participante comme renforçant la distinction entre « vrais » et « faux » réfugiés :

Il y a maintenant une distinction entre les pays d'origine désignée " sûrs », ce qui fait en sorte que tout d'un coup il y a une catégorie de « faux demandeurs d'asile » qui essaient juste de profiter du Canada. Les demandeurs d'asile de ces pays-là c'est presque certain que leur demande va être refusée. Et parmi ces pays-là, ça m'a beaucoup choquée d'apprendre ça, c'est le Mexique. Et pourtant on sait qu'il y a mille et une raisons de fuir ce pays-là. (entrevue 4)

Ainsi, cette distinction est susceptible d'affecter négativement les personnes issues de pays « sûrs », d'autant plus que l'inscription de certains pays dans cette liste paraît douteuse.

Par ailleurs, la réduction des délais avant l'audience constitue le principal élément de la loi C-31 abordé par les participants. Cette réduction des délais semble l'unique aspect des politiques fédérales perçu positivement - avec nuances - par certains participants. En effet, l'incertitude prolongée quant à la possibilité de pouvoir rester au Canada aurait un impact néfaste sur la santé psychologique du demandeur d'asile, notamment à travers le stress et la colère qu'elle provoque, tel que l'explique ce participant :

6. En raison des contraintes d'espace et de l'objet principal de l'article, nous ne nous sommes pas attardés à décrire les besoins identifiés. 
Plus on attend longtemps pour une réponse positive ou négative, il y a une colère qui s'installe chez ces personnes-là, une espèce de rage qui s'installe. Moi, je le vivrai jamais, mais ne pas savoir ce qui va se passer avec moi demain c'est difficile. Donc plus on écourte ce délai-là, que la réponse soit positive ou non, mieux c'est. Par contre, l'idée du deux mois, c'est beaucoup trop rapide, six mois auraient été l'idéal. (entrevue 5)

Ainsi, écourter les délais d'attente semble bénéfique pour certaines personnes qui voient leur situation régulée et qui peuvent ainsi plus rapidement commencer leur vie au Canada. Cela dit, plusieurs participants déplorent le fait que le processus est maintenant beaucoup trop rapide pour la plupart des demandeurs d'asile, qui n'ont pas suffisamment de temps pour se poser au Canada, se préparer à l'audience et recueillir les preuves suffisantes sur leur vécu dans le pays d'origine. Ainsi, ce passage d'un extrême à l'autre - les délais étant passés d'environ un an et demi à deux mois - peut affecter négativement la trajectoire du demandeur d'asile après son arrivée, le forçant à monter son dossier en peu de temps alors qu'il n'est pas nécessairement prêt psychologiquement. Au niveau pratique, ce court délai risque également d'avoir un impact sur la décision rendue par rapport au statut :

Ça [la réduction des délais] a ses avantages pour les gens qui ont beaucoup de preuves, ne sont pas affectés psychologiquement ou pas au point d'être vraiment dysfonctionnels, parce qu'effectivement ça va beaucoup plus vite. Donc pour ces gens-là c'est bien d'avoir une réponse rapide, de pas avoir cette insécurité par rapport à son statut, de savoir où va son avenir. Oui c'est bien pour ça, mais pour les autres personnes - ce qui est probablement la majorité - c'est des gens qui arrivent ici qui sont dysfonctionnels - justement compte tenu de leur vécu - donc qui sont pas prêts encore à être devant un juge, de raconter leur histoire à l'intérieur de deux mois. Des fois ils sont même pas capables d'exprimer leur histoire. Et là, à l'intérieur de deux mois ils sont censés aller devant un juge avec des preuves, êtres concrets, être clairs, être précis au niveau de tout ce qui leur est arrivé. (entrevue 8)

\section{Critique des modifications au Programme fédéral de santé intérimaire}

Plusieurs critiques sont également manifestées par rapport aux modifications du Programme fédéral de santé intérimaire (PFSI) mises en œuvre en juin 2012. Les participants dénoncent notamment l'arrêt de couverture des soins médicaux pour les gens issus des pays d'origine désignés (POD), de même que la réduction de la diversité de services médicaux offerts auparavant à tous les demandeurs d'asile, qui n'a pas été rétablie dans les changements de novembre 2014. Plusieurs participants déplorent en effet l'arrêt de couverture des soins psychologiques offerts aux demandeurs d'asile, considérant qu'il s'agit d'une aide essentielle pour certains d'entre eux à travers le processus d'adaptation. Les propos d'un participant reflètent bien cette incompréhension face aux coupures dans des services cruciaux pour l'adaptation d'un demandeur d'asile après son arrivée, autant sur les plans physique que psychologique :

Les coupures qu'on a vécues... pour le programme de santé fédéral... j'arrive même pas avec une certaine distance à comprendre la pensée en arrière de ça, de carrément éliminer les choses comme les médicaments, les aides à la mobilité, les cannes, les prothèses aux gens qui ont perdu les jambes et les bras, les soins 
dentaires, les lunettes et tout ça, l'aide psychologique pour les personnes torturées, de dire que ça c'est une aide gold plated [...] j'arrive pas à comprendre l'attitude qui peut donner naissance à cette perception-là. (entrevue 3)

Ainsi, les modifications au PFSI sont perçues comme ayant une forte influence sur la santé psychologique des demandeurs d'asile. Les participants ont abordé la manière dont cette réforme affectait les services offerts par leur organisation respective, de même qu'à Montréal de manière générale. Finalement, ils ont manifesté leurs préoccupations face à l'impact de ces coupures sur le vécu des demandeurs d'asile.

\section{Impacts de l'arrêt de couverture des soins psychologiques sur l'offre de services des organisations rencontrées}

Nous nous sommes particulièrement attardés à l'impact de l'arrêt de couverture des soins psychologiques sur le fonctionnement des organisations rencontrées au cours de la recherche. D'abord, cette partie spécifique de la réforme du PFSI a eu un impact nettement plus important sur une organisation, compte tenu de la nature des services d'ordre psychothérapeutiques qu'elle dispensait. Cela dit, plusieurs autres organisations utilisaient cet organisme comme service de référence pour les demandeurs d'asile et ont ainsi vu leur fonctionnement affecté par cette coupure.

\section{Obligation pour une ressource de réduire son mandat et son offre de service}

D'abord, l'organisme le plus touché par l'arrêt de couverture des soins psychologiques s'est vu obligé de réduire son mandat pour correspondre à la mission de leur principale source de fonds, maintenant que le fédéral n'offre plus de remboursement. De plus, les services de soutien thérapeutique dispensés par cette ressource sont désormais limités dans le temps, faute de moyens pour faire un suivi plus approfondi :

C'est ce qu'on offre, un accompagnement personnel, sur une base malheureusement assez limitée ces jours-ci. À cause des coupures, ça se limite à une vingtaine d'heures de thérapie, comparé aux années précédentes où on suivait les gens presque aussi longtemps que nécessaire parce que les traumas sont si importants. Dans le passé on était capable de les suivre selon le vrai besoin de la personne. Maintenant malheureusement ça s'est limité à une vingtaine d'heures, simplement pour des raisons financières, simplement. (entrevue 3 )

Ainsi, en plus de devoir refuser d'activer certains dossiers, cette ressource se voit obligée de réduire le soutien apporté à sa clientèle, malgré un besoin criant pour une partie de celle-ci qui présente des difficultés importantes.

\section{Limitation des services d'aide psychologique offerts}

Quant aux autres organisations pour lesquelles l'arrêt de couverture des soins psychologiques n'a pas eu d'impact sur le financement, plusieurs ont tout de même été affectées par la réforme, principalement, car elles ne pouvaient plus diriger les demandeurs d'asile rencontrés vers la ressource d'aide psychologique qui a dû réduire son mandat. Cette limite a différentes 
conséquences pour ces organisations, car elle engendre un surplus de travail, et que leurs intervenants ne sont pas toujours qualifiés pour offrir des soins psychologiques spécialisés.

\section{Surplus de travail pour les organisations}

D'abord, plusieurs participants ont relaté une augmentation de leur charge de travail sur le plan de l'accompagnement psychologique, vu que les possibilités d'en référer à une aide spécialisée sont maintenant limitées :

Nous, ce qu'on doit faire, c'est qu'on doit redoubler d'effort pour pouvoir offrir à notre manière à nous ces services-là. Dans notre contexte à nous, on est très peu d'employés. En fait je suis le seul employé ici. On doit redoubler d'efforts puis un peu pallier l'absence de services officiels. Donc c'est une augmentation de la charge de travail, ça, c'est clair pour nous. (entrevue 5)

\section{Difficulté à offrir des soins spécialisés en psychologie}

Outre le fait d'avoir à répondre à davantage de besoins des demandeurs d'asile rencontrés, plusieurs participants ont exprimé ne pas avoir les outils nécessaires pour assurer des suivis spécialisés :

Quand j'avais des personnes devant moi qui avaient plus besoin de soins psychologiques, nous on est pas formés pour, nous on n'a pas les outils. Donc l'impact c'est qu'aujourd'hui on a des cas, on sait pas trop comment les aider. On les amène où ? Oui, ça a un impact très négatif parce qu'il y en a pas d'autres. Nous les organismes communautaires on fait pas du soutien psychologique, et donc oui c'est sûr que ça joue sur le moral [...] Bon, nous, le soutien, directement c'était de pouvoir lui dire «bon, ben, tu vas te trouver un réseau », mais, psychologique, je pouvais l'envoyer nulle part hélas, je pouvais l'envoyer nulle part. (entrevue 6)

Ainsi, plutôt que de se concentrer exclusivement sur l'accompagnement psychosocial tel que l'implique leur rôle dans l'organisation, plusieurs participants ont exprimé la préoccupation de devoir assumer un rôle de soutien thérapeutique pour lequel ils ne se sentent pas nécessairement qualifiés. Cela dit, par souci du bien-être de leurs clients, plusieurs intervenants assurent tout de même ce type de soutien :

On n'est pas psychologues et on le sera jamais, mais ça nous incite un peu plus peut-être à développer au niveau de la thérapie, parce qu'il y a pas de ressources en ce moment disponibles, nécessairement donc on est plus portés justement à intervenir peut-être même si on devrait pas intervenir, parce qu'on veut aider la personne puis parce qu'il y a pas accès ailleurs [...]. (entrevue 8)

Bref, bien que l'arrêt de couverture des soins psychologiques ait affecté considérablement une ressource, il a également un impact sur le fonctionnement d'autres ressources qui travaillaient en collaboration avec celle-ci. 


\section{Surcharge des organisations face à l'insuffisance des services gouvernementaux}

Dans un contexte plus large que celui de leur propre organisation, les participants se sont également exprimés sur les impacts de la réforme sur le fonctionnement des services d'aide psychologique à Montréal. Une impression semble répandue chez plusieurs participants, soit la surcharge de travail des organisations face à l'insuffisance des services gouvernementaux:

Habituellement quand il y a une coupure dans une partie des services officiels gouvernementaux, il revient au secteur communautaire de gérer ça [...] Quelqu'un vient en état de crise, nous perdons l'outil pour l'orienter vers une psychothérapie. Donc tout ce qu'on peut faire est faire de notre mieux pour gérer ça [...] Donc ça veut dire que le secteur communautaire doit gérer les conséquences, essayer d'assister les gens avec entre autres un état psychologique de désorganisation. (entrevue 2, traduction libre)

L'augmentation de la charge de travail des organisations sur le plan des services psychologiques s'explique non seulement par les coupures au PFSI, mais également par la conjoncture actuelle de l'offre de services aux demandeurs d'asile. Même si les demandeurs d'asile ont officiellement accès aux mêmes services du CLSC que l'ensemble de la population, l'attente pour obtenir des services psychologiques est longue et le processus parfois complexe :

Maintenant, c'est beaucoup plus difficile parce que justement on n'a pas accès à ces services-là, et on se retrouve dans des situations où on doit référer ces personnes-là dans des CLSC. Déjà c'était difficile au début parce que les CLSC connaissent pas nécessairement les demandeurs d'asile, ils connaissent pas qu'est-ce qui leur est offert, qu'est-ce qui est payé, qu'est-ce qui est pas payé, donc déjà, je te dirais la voie de contact était difficile. En plus y'a des listes d'attente comme pour n'importe qui, en fait. Qu'ils soient citoyens ou demandeurs d'asile, des listes d'attente, ça existe dans le système public, donc c'est beaucoup plus difficile d'avoir accès à ça. (entrevue 8)

Finalement, un participant mentionne la difficulté d'assurer aux demandeurs d'asile un accès aux psychologues du secteur privé, car ces derniers ne seront pas remboursés par le fédéral :

Il y a certains médecins qui refusent d'accepter un demandeur d'asile avec le nouveau PFSI, et sûrement il doit y avoir des psychologues qui font la même chose parce qu'ils sont pas couverts pour leurs consultations ou leur service. Donc voilà, c'est les coupures du gouvernement fédéral qui se répercutent avec les médecins, les psychologues, tous ceux qui donnent les soins de santé. (entrevue 7)

Bref, au-delà des perturbations amenées par l'arrêt de couverture des soins psychologiques au sein des organisations rencontrées, c'est tout le fonctionnement du réseau d'offre de services qui est affecté. 


\section{Impacts de l'arrêt de couverture des soins psychologiques sur le vécu des demandeurs d'asile}

Plusieurs participants ont souligné les impacts de l'arrêt de couverture des soins psychologique sur le vécu des demandeurs d'asile, notamment au niveau de la santé mentale :

Pour beaucoup de personnes, ça aide d'avoir quelqu'un puis ça aide de pouvoir parler de son vécu, pis ça aide d'avoir une réflexion par rapport à ce qu'on a vécu. Donc de pas avoir accès à ce service-là, ça nuit au fonctionnement de la personne, surtout si c'est une personne qui voudrait. Parce que c'est sûr que comme pour n'importe qui, y'a certaines personnes qui veulent recevoir des services et y'en a d'autres qui sont pas prêts à en recevoir. Mais pour les personnes qui sont prêtes à en recevoir un service qui va les aider, ne pas avoir accès à ce service-là... Je crois que c'est un droit universel que tout le monde devrait avoir, puis ces gens-là sont brimés dans leur droit. (entrevue 8)

Ainsi, pour ceux qui ont besoin et qui désirent recevoir des services spécialisés par rapport aux difficultés rencontrées, plusieurs préoccupations émergent. D'abord, l'aggravation des difficultés psychologiques pour une personne qui ne reçoit pas les services à temps est mentionnée : «Le fait de ne plus pouvoir avoir un accès aussi facile qu'avant, ça fait beaucoup de problèmes au niveau psychologique. Ça peut peut-être aller en empirant simplement parce qu'ils ont pas pu avoir accès à une ressource pour tenter de gérer ce qu'ils vivent » (entrevue 4). De plus, le fait de ne pas recevoir de services psychologiques appropriés peut entraver le fonctionnement d'une personne dans d'autres sphères du vécu post-migratoire, tel que l'explique cette participante :

À la fin, ça nuit à leur fonctionnement parce que c'est des gens que, s'ils recevaient leur service, ils pourraient justement éventuellement devenir plus fonctionnels, pouvoir travailler. Se sentir aussi comme quelqu'un qui bénéficie à la société ou qui contribue, donc c'est sûr et certain que ça les affecte, sans aucun doute. (entrevue 8)

Finalement, une participante exprime les émotions difficiles pouvant découler d'un contexte politique qui minimise les besoins des demandeurs d'asile en terme de services psychologiques:

Le fait que ça, ça ait été coupé, c'est comme dire que tout d'un coup ces personneslà ne sont pas importantes. C'est comme si le gouvernement, la population canadienne, les abandonnait quand elles en auraient le plus besoin. Je pense que ça aussi c'est très démoralisant, très dévalorisant, ça doit rajouter un plus grand stress au point de vue psychologique. (entrevue 4)

Ainsi, l'arrêt de couverture des soins psychologiques semble avoir des implications spécifiques sur l'adaptation des demandeurs d'asile, en plus des impacts néfastes du contexte dans laquelle elle s'inscrit.

Bref, la réforme du PFSI est perçue comme ayant un impact majeur sur la santé psychologique des demandeurs d'asile à Montréal. Cet impact s'exerce à différents niveaux, autant sur le fonctionnement des ressources qui en sont affectées que pour la personne qui en subit les 
conséquences après son arrivée au Canada. En plus des modifications à la couverture des soins de santé, nous nous sommes attardés à l'arrêt de couverture des soins psychologiques. Cette partie de la réforme a des implications importantes pour les participants rencontrés, affectant négativement le fonctionnement de plusieurs organisations, mais également la fourniture de services offerts à une population qui présente des besoins psychologiques particuliers.

En somme, au niveau des politiques fédérales, les participants expriment le besoin d'un virage complet, autant sur le plan des orientations qu'au niveau des politiques qui en découlent. Cela dit, ce changement radical risque d'être difficile à effectuer dans le contexte actuel où le gouvernement a imposé des réformes avec une grande rigidité, tel que le mentionne un participant :

De ce gouvernement j'ai aucune attente à ce qu'ils arrivent à mettre un peu d'équilibre dans leur position face aux demandeurs d'asile. Mais pour un autre gouvernement, aux prochaines élections, si on a un autre groupe qui prend la direction du gouvernement... Comme j'ai dit, au moins de rétablir un programme sain qui reflète un peu ce qu'on avait avant les coupures. Mais par rapport à ce gouvernement, j'ai aucun espoir [...] Moi, je mettrais mes énergies plutôt dans les autres gouvernements en attente, de travailler sur leur positionnement pour leur faire comprendre que si jamais ils prennent les rênes du pouvoir qu'ils soient prêts à prendre la responsabilité nécessaire pour changer l'environnement pour les demandeurs d'asile. Le gouvernement actuel c'est une cause perdue. (entrevue 3)

Ainsi, compte tenu des orientations idéologiques qui sous-tendent l'ensemble des actions du gouvernement actuel, l'idée de cibler d'autres partis pour faire la promotion de politiques qui pourraient promouvoir le bien-être des demandeurs d'asile est proposée.

\section{Discussion}

À la lumière de ces résultats, plusieurs aspects propres à la trajectoire psychologique des demandeurs d'asile peuvent être mis en relation avec des facteurs structurels reliés au contexte canadien. 


\section{Barrières à la santé psychologique dans l'ensemble des sphères d'évolution dans la société d'accueil}

Barrières rencontrées par le demandeur d'asile dans le vécu post-migratoire

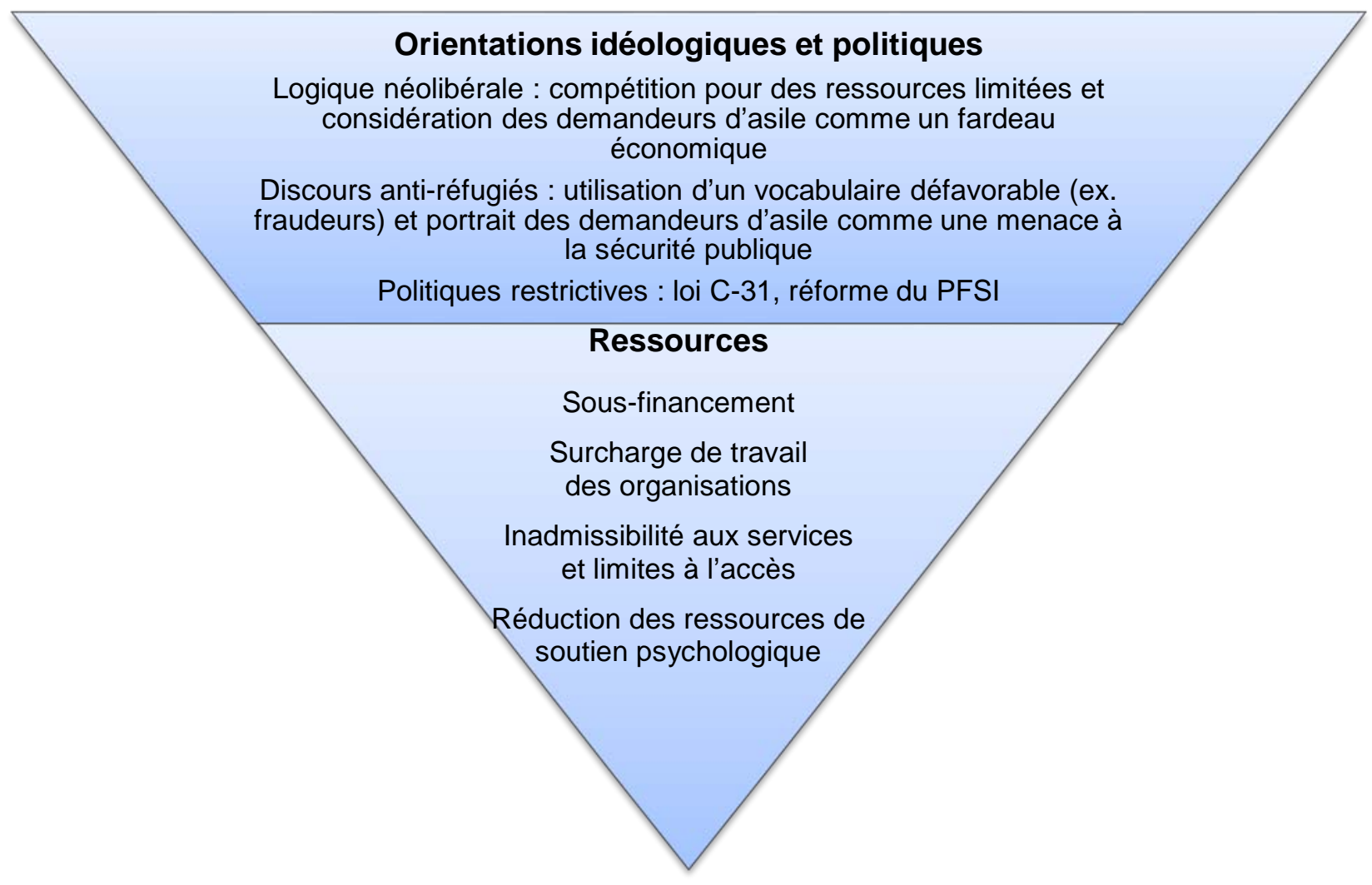

Plusieurs barrières aux besoins des demandeurs d'asile sont présentes dans la société d'accueil, ce qui est d'autant plus préoccupant considérant le lien établi entre les difficultés postmigratoires et l'aggravation des problèmes vécus (Lacroix, 2006). En effet, le manque d'accompagnement et d'intégration dans la société d'accueil semble amplifier les difficultés inhérentes au processus de demande d'asile. Cela dit, ces failles ne s'inscrivent pas uniquement dans un contexte d'intervention individuelle, mais doivent se comprendre dans une perspective globale tenant compte des idéologies socioéconomiques dominantes. Ces idéologies se traduisent dans des orientations politiques affectant le bien-être psychologique des demandeurs d'asile dans l'ensemble de leur cheminement. En effet, ces facteurs structurels affectent non seulement les politiques et les lois à l'égard de cette population, mais également le rapport entre cette dernière et la société d'accueil, ainsi que l'ensemble du fonctionnement des ressources visant à répondre à ses besoins. C'est donc selon cette perspective globale que nous avons exploré ces interactions dans le contexte canadien, plus précisément au Québec.

\section{Idéologie et orientations politiques}

D'abord, la perception des demandeurs d'asile comme une menace à la sécurité et comme une atteinte à la prospérité économique du pays (Okipikti et Aymer, 2003 ; Kissoon, 2010) a été 
largement relevée par les participants rencontrés. Selon les participants de l'étude, ces réformes ont également engendré une diminution du nombre de demandeurs d'asile, reflétant le désengagement de l'État canadien dans l'aide apportée aux réfugiés à l'échelle internationale. En ce sens, une diminution de moitié du nombre de demandeurs d'asile est rapportée par un rapport du Haut Commissariat des Nations unies pour les réfugiés, selon lequel le nombre de demandeurs d'asile serait passé de 20500 en 2012 à 10400 en 2013 (HCR, 2014).

Au niveau de la réduction des délais avant l'audience des demandeurs d'asile (CIC, 2012b), il semblerait que ce changement puisse être bénéfique, considérant les impacts négatifs d'une incertitude prolongée par rapport au statut sur la santé psychologique des demandeurs d'asile dans la société d'accueil (Lacroix, 2004a ; Vignal et Geny-Benkorichi, 2012 ; Bala, 2005). Cela dit, selon les participants, nous sommes maintenant passés à un autre extrême où les courts délais rendent difficiles la collecte de preuves nécessaires à l'obtention du statut de réfugié, de même que la stabilisation de l'état psychologique nécessaire au processus éprouvant que constitue l'audience.

\section{Au niveau des ressources}

La province de Québec n'a pas pallié l'arrêt de couverture des soins psychologiques aux demandeurs d'asile, les services sociaux publics étant théoriquement accessibles à cette population (Gouvernement du Québec, 2013). Ainsi, plusieurs ressources ont directement ou indirectement été affectées par cette coupure spécifique, notamment une ressource spécialisée au niveau du soutien thérapeutique ainsi que d'autres organisations qui pouvaient auparavant diriger leurs clients vers cette ressource, ajoutant ainsi certaines difficultés à leur travail. Cette dynamique reflète bien la surcharge des organisations qui découle du désengagement de l'État à assurer les services nécessaires au bien-être des demandeurs d'asile, désengagement qui se manifeste notamment par une insuffisance des fonds alloués à ces ressources (Oxman-Martinez et al., 2007).

Cela dit, il est essentiel de mentionner l'effort entrepris par les ressources rencontrées pour offrir des services de qualité aux demandeurs d'asile avec peu de moyens, parfois même lorsque cette population tombe hors de leur mandat. Ainsi, plusieurs ressources aident les demandeurs d'asile malgré le fait qu'elles ne reçoivent pas les fonds du Québec pour cette population. Nous avons également pu observer un effort concerté pour faire valoir les droits des demandeurs d'asile auprès de la population et des instances décisionnelles.

\section{CONCLUSION ET RECOMMANDATIONS}

Ce projet avait pour but d'explorer l'impact des orientations politiques actuelles sur la santé psychologique des demandeurs d'asile, notamment en ce qui a trait aux réformes entreprises par les Conservateurs depuis 2011. Des barrières s'exercent à tous les paliers de l'environnement post-migratoire rencontré par les demandeurs d'asile, notamment dans les ressources d'aide qui leur sont destinées ou qui pourraient être développées. En effet, les structures idéologiques et politiques se sont révélées indissociables des facteurs promouvant l'intégration socioéconomique et le bien-être psychologique des demandeurs d'asile, de même que du fonctionnement des ressources allant dans ce sens. 
Nous devons mentionner quelques limites à ce projet de recherche. D'abord, les entrevues effectuées sont d'un nombre limité et reflètent la perception d'informateurs clés dans le contexte montréalais, ce qui limite la généralisation des résultats. En outre, les demandeurs d'asile n'ont pas joué un rôle actif dans l'étude, alors qu'il serait indispensable que ces derniers puissent prendre la parole par rapport aux enjeux importants les concernant, d'autant plus que les notions reliées à la santé psychologique sont complexes et largement dépendantes de facteurs culturels et relatifs à chacun. Tel que reflété dans une étude de Martin, Lapalme et Gutman (2013) auprès de demandeurs d'asile mexicains à Montréal, l'expression des demandeurs d'asile sur leur situation est susceptible de générer plusieurs réflexions sur la réalité de leur vécu subjectif et sur la signification de leur migration, parfois en contradiction avec ce qui est perçu et représenté d'un point de vue extérieur.

À la lumière des résultats, plusieurs recommandations peuvent être effectuées quant à la réponse aux besoins des demandeurs d'asile sur le plan psychologique. D'une part, il semble essentiel de modifier les lois et les politiques sociales à l'égard des demandeurs d'asile afin qu'elles répondent davantage à leurs droits et à leurs besoins. D'abord, le retrait de la liste de pays d'origine désignés s'impose, considérant que cette distinction est marginalisante, accueillant certains demandeurs d'asile avec un préjugé défavorable sur la légitimité de leur demande. De plus, les délais avant l'audience devraient être ajustés de manière à ce que les demandeurs d'asile puissent, si nécessaire, se rétablir psychologiquement et recueillir les preuves et les documents nécessaires à leur requête. Ainsi, sans retomber dans l'ancien système où la personne était plongée dans une incertitude prolongée quant à son statut, il convient de trouver un équilibre pour répondre aux besoins de chacun. Tel que mentionné dans la recherche et dans la réponse des institutions montréalaises à la loi C-31 (Centre de santé et de services sociaux de la Montagne/Programme régional d'accueil et d'intégration des demandeurs d'asile, 2012), un délai de six mois serait idéal. Une autre modification importante mentionnée par les participants de l'étude est la nécessité de remettre en place le Programme fédéral de santé intérimaire comme il fonctionnait avant juin 2012, respectant le droit aux soins de santé de l'ensemble des demandeurs d'asile. Cette recommandation est reliée à la liste de pays d'origine désignés, car les personnes issues de ces pays sont principalement touchées par la réforme ; bien que la décision de novembre 2014 aille dans ce sens, la situation reste à suivre. II convient également de remettre en place les services ayant été coupés pour l'ensemble des demandeurs d'asile, tels que la couverture des consultations psychologiques, considérant l'influence bénéfique de cellesci pour l'adaptation de certains demandeurs d'asile qui en manifestent le besoin.

II est également important d'investir adéquatement dans les ressources d'aide aux demandeurs d'asile, que ce soit au niveau du soutien psychologique, de l'accompagnement ou des ressources d'aide à l'intégration socioéconomique. Cet investissement peut s'effectuer à travers un financement adéquat des organismes communautaires comme des ressources institutionnelles. En effet, les organismes doivent recevoir les fonds nécessaires à l'offre adéquate de leurs services. Un financement suffisant pourrait les aider à répondre aux besoins de l'ensemble de la clientèle desservie dans la pratique, ce qui est susceptible d'inclure des demandeurs d'asile. En outre, bien que des ressources institutionnelles soient également accessibles aux demandeurs d'asile, il est primordial de leur assurer l'accès à ces ressources. 
Pour viser l'atteinte de ces objectifs, il serait pertinent de développer des initiatives de sensibilisation auprès des différents acteurs influents dans le vécu post-migratoire des demandeurs d'asile. D'une part, les intervenants de l'ensemble des milieux susceptibles de rencontrer des demandeurs d'asile doivent être sensibilisés aux enjeux particuliers à cette population, ainsi qu'à ses droits. Au niveau des services psychosociaux, le développement de connaissances propres aux enjeux spécifiques reliés aux demandeurs d'asile et aux réfugiés pourrait permettre un accompagnement adapté à leurs besoins et empreint de sensibilité culturelle. De plus, la sensibilisation des intervenants peut se traduire par des mobilisations pour atteindre le niveau politique et la population, renforçant l'action anti-oppressive essentielle aux secteurs de la santé et des services sociaux et dont l'insuffisance a largement été critiquée (Cemlyn et Briskman, 2003 ; Hill et al., 2009). En outre, les propos des participants de l'étude révèlent une nécessité de contrer les préjugés et la perception défavorable des demandeurs d'asile dans la population. Ainsi, une perception plus adéquate de la réalité des demandeurs d'asile, axée sur leur résilience, peut être promue de différentes façons, par exemple à travers les médias et à travers des campagnes de sensibilisation et d'information.

Finalement, au-delà des recommandations décrites ci-dessus, il nous semble indispensable de viser des changements durables au niveau des fondements de la perception des demandeurs d'asile au Canada. Ainsi, il serait favorable de les percevoir comme des personnes dont les droits humains fondamentaux doivent être respectés et qui peuvent jouer un rôle constructif dans le développement du pays, plutôt que comme une menace à la sécurité et aux ressources de celuici. Ce virage s'inscrit également dans une remise en question de l'idéologie néolibérale dominante dans les pays industrialisés, où la conception de la valeur des êtres humains en termes économiques est valorisée et où les intérêts marchands priment sur les considérations humanitaires.

\section{SARRADET, Sandrine}

Travailleuse sociale

Maîtrise en travail social, Université McGill (2013)

\section{RÉFÉRENCES}

ASFC (Agence des services frontaliers du Canada) (2010). Programme de détention et de renvoi de

ASFC: étude d'évaluation : <http://www.cbsa-asfc.gc.ca/agency-agence/reports-rapports/aeve/2010/dr-rd-fra.html>.

Association du Barreau canadien (2012). Projet de loi C-31 : Loi visant à protéger le système d'immigration du Canada. <http://www.cba.org/ABC/memoires/PDF/12-27-fr.pdf>.

Barreau du Québec (2012). Projet de loi C-31- Loi modifiant la Loi sur l'immigration et la protection des réfugiés, la Loi sur des mesures de réforme équitables concernant les réfugiés, la Loi sur la sûreté du transport maritime et la Loi sur le ministère de la Citoyenneté et de I'Immigration. <http://www.barreau.qc.ca/pdf/medias/positions/2012/20120528-pl-c31.pdf>. 
Bala, J. (2005). « Beyond the Personal Pain: Integrating Social and Political Concerns in Therapy with Refugees », dans D. Ingleby (dir.), Forced Migrations and Mental Health: Rethinking the Care of Refugees and Displaced Persons, New York, Springer, p. 169-183.

Behnia, B. (2003). «Refugees' Convoy of Social Support », International Journal of Mental Health, vol. 32, no 4, p. 6-19.

Bernhard, J.K., L. Goldring, J. Young, C. Berinstein et B. Wilson (2007). « Living with Precarious Legal Status in Canada: Implications for the Well-Being of Children and Families ». Refuge, vol. 24, no 2, p. 101-114.

Briskman, L., et S. Cemlyn (2005). « Reclaiming Humanity for Asylum-Seekers: A Social Work Response », International Social Work, vol. 48, no 6, p. 714-724.

Cambridge, P., et L. Williams (2004). «Approaches to Advocacy for Refugees and Asylum Seekers: A Development Case Study for a Local Support and Advice Service », Journal of Refugee Studies, vol. 17, no 1, p. 97-113.

Carswell, K., P. Blackburn et C. Barker (2011). «The Relationship Between Trauma, PostMigration Problems and the Psychological Well-Being of Refugees and Asylum Seekers », International Journal of Social Psychiatry, vol. 57, no 2, p. 107-119.

Cemlyn, S., et L. Briskman (2003). «Asylum, Children's Rights and Social Work »,Child and Family Social Work, vol. 8, no 3, p. 163-178.

Centre de santé et de services sociaux de la Montagne/Programme régional d'accueil et d'intégration des demandeurs d'asile (2012). Mémoire concernant le projet de loi C-31 présenté au Comité permanent de la citoyenneté et de l'immigration. <http://www.csssdelamontagne.qc.ca/fileadmin/csss_dlm/Publications/MEMOIRE_SUR_C31.FR.CSSSDLM_PRAIDA.mai_2012.pdf>.

CIC (Citoyenneté et Immigration Canada) (2014). Programme fédéral de santé intérimaire: résumé des prestations offertes. <http://www.cic.gc.ca/francais/refugies/exterieur/resumepfsi.asp>.

CIC (Citoyenneté et Immigration Canada) (2012a). Soins de santé - Réfugiés. <http://www.cic.gc.ca/francais/refugies/exterieur/arrivee-sante.asp>.

CIC (Citoyenneté et Immigration Canada) (2012b). Le système canadien d'octroi de l'asile. $<$ http://www.cic.gc.ca/francais/refugies/canada.asp>.

Cleveland, J., C. Rousseau et R. Kronick (2012). Bill C-4: The Impact of Detention and Temporary Status on Asylum Seekers' Mental Health. January 2012 - Brief for submission to the House of Commons Committee on Bill C-4, the Preventing Human Smugglers from Abusing Canada's Immigration System Act :

<http://www.csssdelamontagne.qc.ca/fileadmin/csss_dlm/Publications/Publications_CRF/Imp act_of_Bill_C4_on_asylum_seeker_mental_health_full.pdf $>$.

CISR (Commission de l'immigration et du statut de réfugié au Canada) (2012). Demandes d'asile. < http://www.irb-cisr.gc.ca/Fra/Pages/index.aspx>. 
CISR (Commission de l'immigration et du statut de réfugié au Canada) (2006). Commission de l'immigration et du statut de réfugié au Canada: un aperçu. Ottawa, Direction des communications.

Conseil canadien pour les réfugiés (2012). Changements au système de détermination du statut de réfugié (C-31). <http://ccrweb.ca/fr/la-reforme-refugies>.

Convention de Genève (1951). <http://www.ofii.fr/IMG/pdf/Convention_de_Geneve.pdf>.

Creswell, J.W. (2013). Qualitative Inquiry and Research Design: Choosing Among Five Approaches. Thousand Oaks (CA), Sage.

Crumlish, N., et K. O'Rourke (2010). « A Systematic Review of Treatments for Post-Traumatic Stress Disorders among Refugees and Asylum Seekers », Journal of Nervous and Mental Disease, vol. 198, no 4, p. 237-251.

Des Rosiers, N., et N. Mendelsohn Aviv (2012). Le projet de loi C-31: Une violation injustifiée des droits des personnes en danger :

<http://ccla.org/wordpress/wp-content/uploads/2012/05/2012-04-30-Fr-pr\%C3\%A9sentationau-Comit\%C3\%A9.pdf>.

George, M. (2010). «A Theoretical Understanding of Refugee Trauma », Clinical Social Work Journal, vol. 38, no 4, p. 379-387.

Gladden, J. (2012). « The Coping Skills of East-African Refugees: A Literature Review », Refugee Survey Quarterly, vol. 31, no 3, p. 177-196.

Gouvernement du Québec (2013). Services offerts aux demandeurs d'asile par le Gouvernement du Québec: [http://www4.gouv.qc.ca/FR/Portail/Citoyens/Evenements/immigrer-auquebec/Pages/services-revendicateurs-statut-refugie.aspx].

HCR (Haut Commissariat des Nations unies pour les réfugiés) (2014). Hausse importante du nombre de demandes d'asile dans les pays industrialisés:

$<$ http://www.unhcr.fr/532b1915c.html>.

Hill, L., J. Stroud, R. Gray et S. Chiripanyanga (2009). « Inter-professional Learning to Prepare Medical and Social Work Students for Practice with Refugees and Asylum Seekers », Social Work Education, vol. 28, no 3, p. 298-308.

Kissoon, P. (2010). « From Persecution to Destitution: A Snapshot of Asylum Seekers' Housing and Settlement Experiences in Canada and the United Kingdom », Journal of Immigrant and Refugee Studies, vol. 8, no 1, p. 4-31.

Lacroix, M. (2004a). «Les demandeurs d'asile au Canada : quelques enjeux pour la pratique du travail social », Service Social, vol. 51, no 1, p. 45-59.

Lacroix, M. (2004b). «Canadian Refugee Policy and the Social Construction of the Refugee Claimant Subjectivity: Understanding Refugeeness », Journal of Refugee Studies, vol. 17, no 2, p. 147-166. 
Lacroix, M. (2006). « Social Work with Asylum Seekers in Canada: The Case for Social Justice », International Social Work, vol. 49, no 1, p. 19-28.

Lacroix, M.,et C. Sabbah (2011). « Posttraumatic Psychological Distress and Resettlement: The Need for a Different Practice in Assisting Refugee Families », Journal of Family Social Work, vol. 14, no 1, p. 43-53.

Lacroix-Sarradet, Sandrine (2013). « Adaptation psychologique post-migratoire des demandeurs d'asile dans un contexte politique défavorable : perspectives d'intervention ». Projet d'études indépendant pour l'obtention de la maîtrise en travail social. Montréal, Université McGill.

Martin, P., A. Lapalme et M.R. Gutman (2013). « Réfugiés et demandeurs d'asile mexicains à Montréal : actes de citoyenneté au sein de l'espace nord-américain ? » ACME: An International E-Journal for Critical Geographies, vol. 12, no 3, p. 603-628.

Masocha, S., et M.K. Simpson (2011). « Developing Mental Health Social Work for Asylum Seekers : A Proposed Model for Practice », Journal of Social Work, vol. 12, no 4, p. 423-443.

Médecins du Monde Canada (2015). Mémoires déposés lors du mandat «Consultations particulières et auditions publiques sur les documents intitulés Vers une nouvelle politique québécoise en matière d'immigration, de diversité et d'inclusion » :

$<$ http://www.assnat.qc.ca/fr/travaux-parlementaires/commissions/CRC/mandats/Mandat26361/memoires-deposes.html>.

Okipikti, T., et C. Aymer (2003). «Social Work with African Refugee Children and Their Families », Child and Family Social Work, vol. 8, no 3, p. 213-222.

Oxman-Martinez, J., E. Jimenez, J. Hanley et I. Bohard (2007). « La dynamique triangulaire dans le processus d'incorporation des demandeurs d'asile, les politiques migratoires et le rôle des organismes communautaires », Refuge, vol. 24, n², p. 76-85.

Parlement du Canada (2012). Projet de loi C-31 :

<http://www.parl.gc.ca/HousePublications/Publication.aspx?Docld=2330485\&Language=f\&M ode $=1>$.

Peterson, M. (2012). L'instant du danger. Réflexions d'un psychanalyste et témoignages sur l'exil forcé. Montréal, Éditions du passage.

Simich, L., M. Beiser, M. Stewart et E. Mwakarimba (2005). « Providing Social Support for Immigrant and Refugees in Canada: Challenges and Directions », Journal of Immigrant and Minority Health, vol. 7, no 4, p. 259-268.

Vignal, M., et M. Geny-Benkorichi (2012). État des lieux national de la prise en charge et de la prise en compte de la santé mentale des réfugiés et demandeurs d'asile au sein du dispositif national d'accueil. Réseau Samdarra: Santé mentale, précarité, demandeurs d'asile et réfugiés en Rhône-Alpes :

$<$ http://www.samdarra.fr/documents/Rapportdesynthese etatdeslieuxnational-ReseauSamdar ra_000.pdf>. 